\title{
On Finite Truncation of Infinite Shot Noise Series Representation of Tempered Stable Laws
}

\author{
JUNICHI IMAI* AND REIICHIRO KAWAI ${ }^{\dagger}$
}

\begin{abstract}
Tempered stable processes are widely used in various fields of application as alternatives with finite second moment and long-range Gaussian behaviors to stable processes. Infinite shot noise series representation is the only exact simulation method for the tempered stable process and has recently attracted attention for simulation use with ever improved computational speed. In this paper, we derive series representations for the tempered stable laws of increasing practical interest through the thinning, rejection, and inverse Lévy measure methods. We make a rigorous comparison among those representations, including the existing one due to [18, 34], in terms of the tail mass of Lévy measures which can be simulated under a common finite truncation scheme. The tail mass are derived in closed form for some representations thanks to various structural properties of the tempered stable laws. We prove that the representation via the inverse Lévy measure method achieves a much faster convergence in truncation to the infinite sum than all the other representations. Numerical results are presented to support our theoretical analysis.

Keywords: infinitely divisible random vector, inverse Lévy measure method, rejection method, sample paths simulation, shot noise method, tempered stable process, thinning method.

2010 Mathematics Subject Classification: 60E07, 65B10, 60B10, 65C10.
\end{abstract}

\section{Introduction}

The class of tempered stable law was first proposed by Tweedie [36]. Its associated Lévy and OrnsteinUhlenbeck processes were studied in Barndorff-Nielsen and Shephard [4] and Rosiński [34]. In particular, Rosiński [34] reveals their featuring properties, such as a stable-like behavior over short intervals, the absolute continuity with respect to its short-range limiting stable subordinator, aggregational Gaussianity and a series representation in closed form. Such processes have been introduced in a variety of applications. In mathematical finance, they were introduced to model asset price dynamics in Carr et al. [7] under the name CGMY model and stochastic volatility in Benth et al. [5] and Carr et al. [8]. They were also used in financial econometrics in [4] and in mathematical biology in Palmer et al. [31], to mention just a few. In statistical physics, stochastic processes with heavy marginal probability tails and still with finite variance have been developed through various different direct truncations of the marginal density function of the stable law. The pioneering work of Mantegan and Stanley [28] is the constitution for the so-called truncated Lévy flights in econophysics. In Koponen [26], the analytic expression for characteristic function of truncated Lévy flights was derived. Multi-scaling properties of truncated Lévy flights was investigated by Nakao [30] for the first time. Arbitrary truncation of Lévy flights was considered in Vinogradov [37]. In Figueiredo

\footnotetext{
Published in Physica A (2011) 390(23-24) 4411-4425.

*Email Address: jimai@ae.keio.ac.jp. Postal Address: Faculty of Science and Technology, Keio University, Yokohama, 2238522, Japan.

${ }^{\dagger}$ Corresponding Author. Email Address: reiichiro.kawai@le.ac.uk. Postal Address: Department of Mathematics, University of Leicester, Leicester LE1 7RH, UK. Tel: +44-116-223-1310. Fax: +44-116-252-3915.
} 
et al. [14], truncated Lévy flights are extended to possess autocorrelation, which is often observed in the real-world financial data. Various other related models were studied in, for instance, Gupta and Campanha [15], Matsushita, Rathie and Da Silva [29], and Podobnik et al. [32]. Those truncated Lévy flight models are adequately capable of describing a variety of stylized properties seen in time series of complex systems and have been appealing due to their intuitive approach, while they are no longer Lévy processes and are thus not robust to the observation time scale. This fact ensure practical importance and sample path generation of tempered stable processes.

Several methods for simulation of sample paths of tempered stable Lévy processes have been investigated in the literature. It is well known (Devroye [12] and Baeumer and Meerschaert [3]) that their univariate increments with stability index smaller than one can be simulated in the exact sense through acceptancerejection sampling using its non-tempered stable random variables as proposal distribution. The case of stability index greater than one is also discussed in [3] and Kawai and Masuda [25] in an approximative sense. With the help of those acceptance-rejection, simulations of tempered stable Ornstein-Uhlenbeck processes are investigated in Kawai and Masuda [23, 24] under a discrete observation setting. The other route of sample paths simulation is based on infinite shot noise series representations (Bondesson [6] and Rosiński [33]). Strictly speaking, the infinite shot noise series is the only exact simulation method for tempered stable processes to realize all the information of sample paths, that is, size, direction and timing of every single jump. A closed form of such a series representation was derived in Rosiński [34] (first introduced in his discussion section of the article [4]). Its structure is sufficiently simple and enables one to simulate sample paths solely with elementary random variables, such as exponential and uniform. From a computational point of view, the form of infinite sum raises issues of heavy computing load and of finite truncation [10] and suggests variance reduction for Monte Carlo simulation purposes [19, 20].

The main purpose of this paper is to investigate a finite truncation of infinite shot noise series representation of the tempered stable law from a numerical standpoint in order to facilitate the simulation use of such infinite series representations. By applying several methods for deriving a kernel of shot noise series (see Rosiński [33]), we derive and present in Section 3 five different representations of the tempered stable law. Our main results are presented in Theorem 3.1 on the finite truncation of those five representations. In short, the inverse Lévy measure method [13, 27] simulates more mass of Lévy measure tails under a common finite truncation scheme, compared to representations based on the rejection and thinning methods [33] and the representation of Rosiński [34]. In connection with recent results of the authors [17, 18], the inverse Lévy measure method is most attractive from a simulation point of view. In the representation through the inverse Lévy measure method, we only need to generate Poisson arrival times for the jump size and a few others for jump timing and direction. In the case of the tempered stable law, however, the tail inverse of Lévy measure is not available in closed form. This issue was addressed in [18] through a numerical approach, which we will review in Appendix $\mathrm{A}$ to keep this paper self-contained. Numerical results on moment estimation of unilateral tempered stable random variable are presented in Section 4 to provide the support for our theoretical analysis. To avoid overloading the paper with rather lengthy proofs of somewhat routine nature, we omit non-essential details in some instances.

\section{Preliminaries}

Let us begin this section with the notations which will be used throughout the paper. We denote by $\mathbb{R}^{d}$ the $d$-dimensional Euclidean space with the norm $\|\cdot\|, \mathbb{R}_{0}^{d}:=\mathbb{R}^{d} \backslash\{0\}, \mathbb{R}_{+}:=(0,+\infty)$ and $\mathscr{B}\left(\mathbb{R}_{0}^{d}\right)$ is the Borel $\sigma$-field of $\mathbb{R}_{0}^{d}$. We let $\mathbb{N}$ be the collection of positive integers, with $\mathbb{N}_{0}:=\mathbb{N} \cup\{0\}$. We denote by $\stackrel{\mathscr{L}}{=}$ and $\stackrel{\mathscr{L}}{\rightarrow}$, respectively, identity and convergence in law. Finally, we define the following.

(i) $\left\{E_{k}\right\}_{k \in \mathbb{N}}$ is a sequence of iid exponential random variables with unit mean, 
(ii) $\quad\left\{\Gamma_{k}\right\}_{k \in \mathbb{N}}$ is a sequence of standard Poisson arrival times, generated iteratively as a successive summation of iid exponential random variables;

$$
\left\{\Gamma_{1}, \Gamma_{2}, \Gamma_{3}, \ldots\right\} \leftarrow\left\{\sum_{k=1}^{1} E_{k}, \sum_{k=1}^{2} E_{k}, \sum_{k=1}^{3} E_{k}, \ldots\right\}
$$

The exponential random variables $\left\{E_{k}\right\}_{k \in \mathbb{N}}$ serve as Poisson interarrival times.

\subsection{Tempered Stable Law}

We here review the tempered stable law defined by Rosiński [34]. An infinitely divisible probability measure $\mu$ on $\mathbb{R}^{d}$, without Gaussian component, is called tempered stable if its Lévy measure has the form

$$
v(B)=\int_{\mathbb{R}_{0}^{d}} \int_{\mathbb{R}_{+}} \mathbb{1}_{B}(r v) \frac{e^{-r}}{r^{\alpha+1}} d r \rho(d v), \quad B \in \mathscr{B}\left(\mathbb{R}_{0}^{d}\right)
$$

where $\alpha \in(0,2)$ and where the measure $\rho$ on $\mathbb{R}_{0}^{d}$ satisfies

$$
\int_{\mathbb{R}_{0}^{d}}\|v\|^{\alpha} \rho(d v)<+\infty .
$$

The two parameters $\alpha$ and $\rho$ uniquely identify Lévy measure of the tempered stable law. Under the additional condition

$$
\begin{cases}\int_{\mathbb{R}_{0}^{d}}\|v\| \rho(d v)<+\infty, & \text { if } \alpha \in(0,1), \\ \int_{\mathbb{R}_{0}^{d}}\|v\|\left(1+\ln ^{+}\|v\|\right) \rho(d v)<+\infty, & \text { if } \alpha=1,\end{cases}
$$

the characteristic function of $\mu$ has a closed form expression given by

$$
\widehat{\mu}(y)=\exp \left[i\langle y, b\rangle+\int_{\mathbb{R}_{0}^{d}} \phi_{\alpha}(\langle y, v\rangle) \rho(d v)\right],
$$

for some $b \in \mathbb{R}^{d}$ and where, for $s \in \mathbb{R}$,

$$
\phi_{\alpha}(s)= \begin{cases}\Gamma(-\alpha)\left((1-i s)^{\alpha}-1+i \alpha s\right), & \text { if } \alpha \in(0,1) \cup(1,2), \\ (1-i s) \ln (1-i s)+i s, & \text { if } \alpha=1 .\end{cases}
$$

For further details about its distributional properties, we refer the reader to [34]. Let us briefly survey known simulation methods for the tempered stable law, except for the one based on infinite shot noise series representation.

(i) The most primitive and direct method for the tempered stable law is based on the use of its probability distribution function computed from the characteristic function (2.5) by the Gil-Pelaez formula. However, we need to deal with numerical integrations for the formula, and also need to invert the distribution for each uniform random variable.

(ii) Gaussian approximation of small jump component was justified by [2, 10], while the remaining component is compound Poisson. Let us clarify that simulation of the compound Poisson component is not as easy as often claimed in the literature, as its standardized distribution function is not available in closed form and the intensity may be extremely large depending on the choice of the small jump component. Great care should thus be taken when addressing this trade-off issue. 
(iii) The one-dimensional unilateral tempered stable distribution admits a density function, which is an exponential tilting of the density of its corresponding non-tempered stable distribution. Together with the well known exact, yet simple, simulation method of Chambers et al. [9], we can apply acceptance-rejection sampling. If its stability index is less than one, then the support of the distribution is half real line and thus the acceptance-rejection sampling method is exact. (See, for example, [3, 12, 23].) Otherwise, the support is the entire real line, while acceptance-rejection sampling can still be applied in an approximative sense due to Baeumer and Meerschaert [3]. (See also [24] for further analysis.)

(iv) Related to (iii), the one-dimensional unilateral distribution can be evaluated for computation of the expectation based on Theorem 33.3 of Sato [35]. In this case, the standardized exponential tilting acts as the Radon-Nykodym derivative for change of measure. This method is exact, regardless of stability index, while is only valid for computation of the expectation, not for sample paths simulation. (See, for example, [22] for practical use of this method.)

To the best of our knowledge, as just described, an exact simulation is possible in the path-wise sense only for the one-dimensional unilateral case with stability index less than one. This observation justifies our investigation of infinite shot noise series representation from a numerical point of view.

\subsection{A Shot Noise Series Representation of Tempered Stable Laws}

Let us first review generalities on the series representation of general infinitely divisible law. Our startup discussion is essentially parallel to the inverse Lévy measure method of [13, 27]. Notice first that the random variable $\sum_{k=1}^{+\infty} \Gamma_{k} \mathbb{1}_{[0, T]}\left(\Gamma_{k}\right)$ is infinitely divisible with Lévy measure $v(d z)=d z$ defined on $(0, T]$. Recall also that the epochs of an inhomogeneous Poisson process on $[0, T]$ with intensity $h(t)$ can be generated by $H\left(\Gamma_{1}\right), H\left(\Gamma_{2}\right), \ldots$, where $H(t)=\inf \left\{u \in[0, T]: \int_{0}^{u} h(s) d s<t\right\}$, provided that $\int_{0}^{T} h(s) d s<+\infty$. Therefore, by regarding the intensity $h(t)$ as a Lévy measure ("on state space" rather than "on time"), we deduce that $\sum_{k=1}^{+\infty} H\left(\Gamma_{k}\right) \mathbb{1}_{[0, T]}\left(\Gamma_{k}\right)$ is an infinitely divisible random variable with Lévy measure $v(d z)=h(z) d z$ defined on $(0, T]$. Notice here that the definition of $H(t)$ implicitly assumes that that the Lévy measure $v$ has a compact support. Moreover, the condition $\int_{0}^{T} h(s) d s<+\infty$ indicates a finite Lévy measure. We can extend this formulation to an infinite Lévy measure on $\mathbb{R}_{+}$, simply by redefining the kernel $H$ as running down from the infinity rather than up the other way, that is, $H(r)=\inf \left\{u \in \mathbb{R}_{+}: \int_{u}^{+\infty} h(s) d s<r\right\}$, and compute $\sum_{k=1}^{+\infty} H\left(\Gamma_{k}\right)$, where $\left\{\Gamma_{k}\right\}_{k \in \mathbb{N}}$ is no longer restricted on a finite interval [0,T]. (See Asmussen and Glynn [1].)

The most general form of series representations is given by the so-called generalized shot noise method introduced by Bondesson [6] and Rosiński [33]. Assume that Lévy measure $v$ defined on $\mathbb{R}_{0}^{d}$ can be decomposed as

$$
v(B)=\int_{\mathbb{R}_{+}} \mathbb{P}(H(r, U) \in B) d r, \quad B \in \mathscr{B}\left(\mathbb{R}_{0}^{d}\right),
$$

where $U$ is a random variate taking values in a suitable space $\mathscr{U}$, and where $H: \mathbb{R}_{+} \times \mathscr{U} \mapsto \mathbb{R}_{0}^{d}$ is such that for each $u \in \mathscr{U}, r \mapsto\|H(r, u)\|$ is non-increasing. Let $\left\{U_{k}\right\}_{k \in \mathbb{N}}$ be a sequence of iid copies of the random variate $U$, independent of $\left\{\Gamma_{k}\right\}_{k \in \mathbb{N}}$. Then, the random vector

$$
\sum_{k=1}^{+\infty}\left(H\left(\Gamma_{k}, U_{k}\right)-\mathbb{E}\left[H\left(\Gamma_{k}, U_{k}\right) \mathbb{1}\left(\left\|H\left(\Gamma_{k}, U_{k}\right)\right\| \leq 1\right)\right]\right)
$$

has an infinitely divisible law with characteristic function

$$
y \mapsto \exp \left[\int_{\mathbb{R}_{0}^{d}}\left(e^{i\langle y, z\rangle}-1-i\langle y, z\rangle \mathbb{1}_{(0,1]}(\|z\|)\right) v(d z)\right] .
$$


A series representation of the tempered stable law is derived in Rosiński [34] through the generalized shot noise method. Let $\left\{W_{k}\right\}_{k \in \mathbb{N}}$ be a sequence of iid standard exponential random variables, let $\left\{U_{k}\right\}_{k \in \mathbb{N}}$ be a sequence of iid uniform random variables on $[0,1]$, let $\left\{V_{k}\right\}_{k \in \mathbb{N}}$ be a sequence of iid random vectors in $\mathbb{R}_{0}^{d}$ with common distribution

$$
\frac{\|v\|^{\alpha} \rho(d v)}{m_{\alpha, \rho}}
$$

with

$$
m_{\alpha, \rho}:=\int_{\mathbb{R}_{0}^{d}}\|v\|^{\alpha} \rho(d v) .
$$

Also, let $k_{0}=\int_{\mathbb{R}_{0}^{d}} v\|v\|^{\alpha-1} \rho(d v) / m_{\alpha, \rho}$, and let

$$
z_{0}:= \begin{cases}\left(\alpha / m_{\alpha, \rho}\right)^{-1 / \alpha} \zeta(1 / \alpha) k_{0}+|\Gamma(1-\alpha)| \int_{\mathbb{R}_{0}^{d}} v \rho(d v), & \text { if } \alpha \neq 1, \\ \left(\alpha^{-1} \ln \left(m_{\alpha, \rho}\right)+2 \gamma\right) \int_{\mathbb{R}_{0}^{d}} v \rho(d v)-\int_{\mathbb{R}_{0}^{d}} v \ln \|v\| \rho(d v), & \text { if } \alpha=1,\end{cases}
$$

where $\zeta$ denotes the Riemann zeta function and $\gamma(=0.5772 \ldots)$ is the Euler constant. Then, by Theorem 5.4 of [34], it is known that the random vector

$$
\sum_{k=1}^{+\infty}\left[\left(\left(\frac{\alpha \Gamma_{k}}{m_{\alpha, \rho}}\right)^{-1 / \alpha} \wedge W_{k} U_{k}^{1 / \alpha}\left\|V_{k}\right\|\right) \frac{V_{k}}{\left\|V_{k}\right\|}-\left(\frac{\alpha k}{m_{\alpha, \rho}}\right)^{-1 / \alpha} k_{0}\right]+z_{0}
$$

has the tempered stable law with characteristic function (2.5) with $b=0$.

\section{Main Results}

In this section, we derive three infinite shot noise series representations of tempered stable law and compare them, including two known representations of [18, 34]. Let us first prepare some notations, which will be used in what follows. Fix $\lambda \in(0,1], \lambda_{1} \in \mathbb{R}_{+}$and $\lambda_{2} \in(0,1]$. Define

$$
\begin{aligned}
& H_{1}(r, w, u, v):=\left[\left(\frac{\alpha r}{m_{\alpha, \rho}}\right)^{-1 / \alpha} \wedge w u^{1 / \alpha}\|v\|\right] \frac{v}{\|v\|}, \\
& H_{2}(r, w, v):=w \mathbb{1}\left(r \leq m_{\alpha, \rho} \frac{\|v\|}{\lambda} \frac{e^{-\frac{1-\lambda}{\|v\|} w}}{w^{\alpha+1}}\right) \frac{v}{\|v\|}, \\
& H_{3}(r, w, v):=w \mathbb{1}\left(r \leq m_{\alpha, \rho} \Gamma\left(\lambda_{1}\right)\left(\frac{\|v\|}{\lambda_{2}}\right)^{\lambda_{1}} \frac{e^{-\frac{1-\lambda_{2}}{\|v\|}}}{w^{\alpha+\lambda_{1}}}\right) \frac{v}{\|v\|}, \\
& H_{4}(r, u, v):=H_{(\mathrm{s})}(r) \mathbb{1}\left(e^{-H_{(\mathrm{s})}(r) /\|v\|}>u\right) \frac{v}{\|v\|}, \\
& H_{5}(r, v):=\inf \left\{u \in \mathbb{R}_{+}: \int_{u}^{+\infty} m_{\alpha, \rho} \frac{e^{-s /\|v\|}}{s^{\alpha+1}} d s>r\right\} \frac{v}{\|v\|} .
\end{aligned}
$$

We will also use the notation

$$
H_{(\mathrm{s})}(r):=\left(\frac{\alpha r}{m_{\alpha, \rho}}\right)^{-1 / \alpha} .
$$

Each of the above serve as a kernel of infinite shot noise series representation. Set the following random sequences. 
(i) $\left\{\Gamma_{k}\right\}_{k \in \mathbb{N}}$ is a sequence of standard Poisson arrival times,

(ii) $\left\{V_{k}\right\}_{k \in \mathbb{N}}$ is a sequence of iid random vectors with common distribution $\|v\|^{\alpha} \rho(d v) / m_{\alpha, \rho}$ on $\mathbb{R}_{0}^{d}$,

(iii) $\left\{U_{k}\right\}_{k \in \mathbb{N}}$ is a sequence of iid uniform random variables on $[0,1]$,

(iv) $\left\{W_{k}^{(1)}\right\}_{k \in \mathbb{N}}$ is a sequence of iid standard exponential random variables,

(v) $\left\{W_{k}^{(2)}\right\}_{k \in \mathbb{N}}$ is a sequence of independent exponential random variables with $W_{k}^{(2)}$ having rate $\lambda /\left\|V_{k}\right\|$ conditionally on $\left\|V_{k}\right\|$,

(vi) $\quad\left\{W_{k}^{(3)}\right\}_{k \in \mathbb{N}}$ is a sequence of independent gamma random variables with $W_{k}^{(3)}$ having shape $\lambda_{1}$ and scale $\left\|V_{k}\right\| / \lambda_{2}$ conditionally on $\left\|V_{k}\right\|$.

As described in Section 2.2, any shot noise series representation of the tempered stable law has the form of infinite sum, since the associated Lévy measure is infinite. To deal with infinite sums in simulation, we need to truncate them up to a certain finite point. A straightforward approach is the truncation to a finite number of terms of the series, that is, $\sum_{k=1}^{n}$ for some $n \in \mathbb{N}$. In this paper, however, we adopt another approach based on the truncation to a finite time span of the underlying standard Poisson process, that is, $\sum_{\left\{k \in \mathbb{N}: \Gamma_{k} \leq n\right\}}$. Clearly, truncation level is left random and is thus different for different replications, while it is almost surely finite. This truncation scheme enables us to trace the part of Lévy measure, which shot noise series can simulate. With this truncation scheme, define for each $n \in \mathbb{N}$,

$$
\begin{aligned}
X_{1, n} & :=\sum_{\left\{k \in \mathbb{N}: \Gamma_{k} \leq n\right\}}\left[H_{1}\left(\Gamma_{k}, W_{k}^{(1)}, U_{k}, V_{k}\right)-c_{1, k}\right], \\
X_{2, n} & :=\sum_{\left\{k \in \mathbb{N}: \Gamma_{k} \leq n\right\}}\left[H_{2}\left(\Gamma_{k}, W_{k}^{(2)}, V_{k}\right)-c_{2, k}\right], \\
X_{3, n} & :=\sum_{\left\{k \in \mathbb{N}: \Gamma_{k} \leq n\right\}}\left[H_{3}\left(\Gamma_{k}, W_{k}^{(3)}, V_{k}\right)-c_{3, k}\right], \\
X_{4, n} & :=\sum_{\left\{k \in \mathbb{N}: \Gamma_{k} \leq n\right\}}\left[H_{4}\left(\Gamma_{k}, U_{k}, V_{k}\right)-c_{4, k}\right], \\
X_{5, n} & :=\sum_{\left\{k \in \mathbb{N}: \Gamma_{k} \leq n\right\}}\left[H_{5}\left(\Gamma_{k}, V_{k}\right)-c_{5, k}\right],
\end{aligned}
$$

where

$$
\begin{aligned}
& c_{1, k}:=\mathbb{E}\left[H_{1}\left(\Gamma_{k}, W_{k}^{(1)}, U_{k}, V_{k}\right) \mathbb{1}_{(0,1]}\left(\left\|H_{1}\left(\Gamma_{k}, W_{k}^{(1)}, U_{k}, V_{k}\right)\right\|\right)\right], \\
& c_{2, k}:=\mathbb{E}\left[H_{2}\left(\Gamma_{k}, W_{k}^{(2)}, V_{k}\right) \mathbb{1}_{(0,1]}\left(\left\|H_{2}\left(\Gamma_{k}, W_{k}^{(2)}, V_{k}\right)\right\|\right)\right], \\
& c_{3, k}:=\mathbb{E}\left[H_{3}\left(\Gamma_{k}, W_{k}^{(3)}, V_{k}\right) \mathbb{1}_{(0,1]}\left(\left\|H_{3}\left(\Gamma_{k}, W_{k}^{(3)}, V_{k}\right)\right\|\right)\right], \\
& c_{4, k}:=\mathbb{E}\left[H_{4}\left(\Gamma_{k}, U_{k}, V_{k}\right) \mathbb{1}_{(0,1]}\left(\left\|H_{4}\left(\Gamma_{k}, U_{k}, V_{k}\right)\right\|\right)\right], \\
& c_{5, k}:=\mathbb{E}\left[H_{5}\left(\Gamma_{k}, V_{k}\right) \mathbb{1}_{(0,1]}\left(\left\|H_{5}\left(\Gamma_{k}, V_{k}\right)\right\|\right)\right] .
\end{aligned}
$$

The representation (3.1) was derived by Rosiński [34] (first introduced in his discussion part of [4]). The representations (3.2) and (3.3) are due to the thinning method of Rosiński [33]. The representation (3.4) is due to the rejection method of Rosiński [33]. The representation (3.5) is based on the inverse Lévy measure method.

Theorem 3.1. (i) For each $k=1,2,3,4,5$, the law $\mathscr{L}\left(X_{k, n}\right)$ is infinitely divisible with Lévy measure $v_{n, k}$ and converges to the infinitely divisible law with triplet $(0,0, v)$ as $n \uparrow+\infty$.

(ii) It holds that for each $n \in \mathbb{N}$,

$$
v_{k, n}\left(\mathbb{R}_{0}^{d}\right)=n, \quad k=1,2,3,4,5 .
$$


(iii) It holds that for each $n \in \mathbb{N}, x \in \mathbb{R}_{+}$and $C \in \mathscr{B}\left(S^{d-1}\right)$,

$$
\begin{aligned}
& v_{1, n}((x,+\infty) C)=\left(n \frac{\alpha}{m_{\alpha, \rho}} x^{\alpha} \wedge 1\right) v((x,+\infty) C), \\
& v_{4, n}((x,+\infty) C)=v\left(\left(\left(\alpha n / m_{\alpha, \rho}\right)^{-1 / \alpha},+\infty\right) C\right) \wedge v((x,+\infty) C), \\
& v_{k, n}((x,+\infty) C) \leq v_{5, n}((x,+\infty) C), \quad k=1,2,3,4
\end{aligned}
$$

(iv) It holds that for each $n \in \mathbb{N}$ and $q \geq 0$ such that $\int_{\|z\|>1}\|z\|^{q} v(d z)<+\infty$,

$$
\int_{\mathbb{R}_{0}^{d}}\|z\|^{q} v_{k, n}(d z)<\int_{\mathbb{R}_{0}^{d}}\|z\|^{q} v_{5, n}(d z) \leq \int_{\mathbb{R}_{0}^{d}}\|z\|^{q} v(d z), \quad k=1,2,3,4 .
$$

First, the result (ii) asserts that all the five representations account for the mass $n$ out of the infinite Lévy measure $v$ when truncating infinite series by $\left\{k \in \mathbb{N}: \Gamma_{k} \leq n\right\}$. The results (iii) provide useful information about the way the representations simulate tails of Lévy measure. In particular, the tail masses (3.6) and (3.7) are available in closed form thanks to various structural properties of the tempered stable laws and show the effect of the almost sure finite truncation scheme $\left\{k \in \mathbb{N}: \Gamma_{k} \leq n\right\}$ for the representations of Rosiński (3.1) and of the rejection method (3.4) in the exact sense. Moreover, the inequality (3.8) indicates that whenever the truncation is performed, the three representations (via the rejection and thinning methods) cannot simulate the tail of the Lévy measure as much as the inverse Lévy measure method. In conjunction with the result (ii), we conclude that the three representations instead simulate some part of the Lévy measure closer to the origin. The inequality (iv) is a direct consequence of this observation. Since this fact holds for any truncation level $n$, the inverse Lévy measure method dominates over the other three representations. It however seems difficult to find clear dominance relations between the representation (3.1) and the others. We will compare those shortly through numerical experiments.

Proof. Throughout the proof, we will use the notations

$$
f(x ; a, b):=\frac{b^{a}}{\Gamma(a)} x^{a-1} e^{-b x}, \quad x \in \mathbb{R}_{+}
$$

for the gamma probability density function with $a>0$ and $b>0$, and

$$
\widetilde{\rho}(d v):=\frac{\|v\|^{\alpha}}{m_{\alpha, \rho}} \rho(d v), \quad v \in \mathbb{R}_{0}^{d} .
$$

Note first that the truncation $\left\{k \in \mathbb{N}: \Gamma_{k} \leq n\right\}$ of infinite series corresponds to the truncation of the integral with respect to the Lebesgue measure $d r$ to the interval $(0, n)$ in the decomposition 2.7).

(i) First of all, a change of variables yields the polar decomposition

$$
v(B)=\int_{\mathbb{R}_{0}^{d}} \int_{\mathbb{R}_{+}} \mathbb{1}_{B}\left(r \frac{v}{\|v\|}\right) m_{\alpha, \rho} \frac{e^{-r /\|v\|}}{r^{\alpha+1}} d r \widetilde{\rho}(d v), \quad B \in \mathscr{B}\left(\mathbb{R}_{0}^{d}\right) .
$$

The variable $v$ in this representation corresponds to the random sequence $\left\{V_{k}\right\}_{k \in \mathbb{N}}$.

We begin with the representation of $\left\{X_{1, n}\right\}_{n \in \mathbb{N}}$. To this end, let us first decompose $v((x,+\infty) C)$. Observe 
that for each $x \in \mathbb{R}_{+}$and $C \in \mathscr{B}\left(S^{d-1}\right)$,

$$
\begin{aligned}
v & ((x,+\infty) C) \\
& =\int_{\mathbb{R}_{0}^{d}} \int_{\mathbb{R}_{+}} \mathbb{1}_{(x,+\infty)}(w\|v\|) \frac{e^{-w}}{w^{\alpha+1}} d w \mathbb{1}_{C}\left(\frac{v}{\|v\|}\right) \rho(d v) \\
& =\int_{\mathbb{R}_{0}^{d}} \int_{x /\|v\|}^{+\infty} \frac{e^{-w}}{w^{\alpha+1}} d w \mathbb{1}_{C}\left(\frac{v}{\|v\|}\right) \rho(d v) \\
& =\int_{\mathbb{R}_{0}^{d}} \frac{1}{\alpha}\left(\left(\frac{x}{\|v\|}\right)^{-\alpha} e^{-x /\|v\|}-\int_{\mathbb{R}_{+}} \mathbb{1}_{(x,+\infty)}(w\|v\|) \frac{e^{-w}}{w^{\alpha}} d w\right) \mathbb{1}_{C}\left(\frac{v}{\|v\|}\right) \rho(d v) \\
& =\int_{\mathbb{R}_{0}^{d}} \int_{\mathbb{R}_{+}} \mathbb{1}_{(x,+\infty)}(w\|v\|) \frac{x^{-\alpha}-(w\|v\|)^{-\alpha}}{\alpha} e^{-w} d w \mathbb{1}_{C}\left(\frac{v}{\|v\|}\right)\|v\|^{\alpha} \rho(d v) \\
& =\int_{\mathbb{R}_{0}^{d}} \int_{\mathbb{R}_{+}} \int_{0}^{1} \mathbb{1}_{(x,+\infty)}\left(w u^{1 / \alpha}\|v\|\right) \frac{m_{\alpha, \rho}}{\alpha} x^{-\alpha} d u e^{-w} d w \mathbb{1}_{C}\left(\frac{v}{\|v\|}\right) \widetilde{\rho}(d v) \\
& =\int_{\mathbb{R}_{0}^{d}} \int_{\mathbb{R}_{+}} \int_{0}^{1} \int_{\mathbb{R}_{+}} \mathbb{1}_{(x,+\infty)}\left(\left(\frac{\alpha r}{m_{\alpha, \rho}}\right)^{-1 / \alpha} \wedge w u^{1 / \alpha}\|v\|\right) d r d u e^{-w} d w \mathbb{1}_{C}\left(\frac{v}{\|v\|}\right) \widetilde{\rho}(d v),
\end{aligned}
$$

where the fifth equality holds by

$$
\begin{aligned}
\mathbb{1}_{(x,+\infty)}(w\|v\|)\left[1-\left(\frac{w\|v\|}{x}\right)^{-\alpha}\right] & =\operatorname{Leb}\left[(0,1) \cap\left(\left(\frac{w\|v\|}{x}\right)^{-\alpha},+\infty\right)\right] \\
& =\int_{0}^{1} \mathbb{1}\left(u^{1 / \alpha}>\frac{x}{w\|v\|}\right) d u
\end{aligned}
$$

while the sixth equality holds by

$$
\begin{aligned}
\mathbb{1}_{(x,+\infty)}\left(w u^{1 / \alpha}\|v\|\right) \frac{m_{\alpha, \rho}}{\alpha} x^{-\alpha} & =\mathbb{1}_{(x,+\infty)}\left(w u^{1 / \alpha}\|v\|\right) \int_{\mathbb{R}_{+}} \mathbb{1}_{(x,+\infty)}\left[\left(\frac{\alpha r}{m_{\alpha, \rho}}\right)^{-1 / \alpha}\right] d r \\
& =\int_{\mathbb{R}_{+}} \mathbb{1}_{(x,+\infty)}\left[\left(\frac{\alpha r}{m_{\alpha, \rho}}\right)^{-1 / \alpha} \wedge w u^{1 / \alpha}\|v\|\right] d r
\end{aligned}
$$

By applying the generalized shot noise method [6, 33], we get the desired series representation.

To prove the claim for $\left\{X_{2, n}\right\}_{n \in \mathbb{N}}$, consider the probability measure on $\mathbb{R}_{0}^{d}$,

$$
F_{1}(B)=\int_{\mathbb{R}_{0}^{d}} \int_{\mathbb{R}_{+}} \mathbb{1}_{B}\left(r \frac{v}{\|v\|}\right) f(r ; 1, \lambda /\|v\|) d r \widetilde{\rho}(d v), \quad B \in \mathscr{B}\left(\mathbb{R}_{0}^{d}\right)
$$

The Lévy measure $v$ is absolutely continuous with respect to $F_{1}$ with Radon-Nykodym derivative

$$
\frac{d v}{d F_{1}}(r, v)=m_{\alpha, \rho} \frac{\|v\|}{\lambda} \frac{e^{-(1-\lambda) r /\|v\|}}{r^{\alpha+1}}, \quad(r, v) \in \mathbb{R}_{+} \times \mathbb{R}_{0}^{d} .
$$

By applying the thinning method [33], we get the desired representation. The proof for $\left\{X_{3, n}\right\}_{n \in \mathbb{N}}$ is similar. Consider the probability measure

$$
F_{2}(B)=\int_{\mathbb{R}_{0}^{d}} \int_{\mathbb{R}_{+}} \mathbb{1}_{B}\left(r \frac{v}{\|v\|}\right) f\left(r ; \lambda_{1}, \lambda_{2} /\|v\|\right) d r \widetilde{\rho}(d v), \quad B \in \mathscr{B}\left(\mathbb{R}_{0}^{d}\right)
$$


The Lévy measure $v$ is absolutely continuous with respect to $F_{2}$ with Radon-Nykodym derivative

$$
\frac{d v}{d F_{2}}(r, v)=m_{\alpha, \rho} \Gamma\left(\lambda_{1}\right)\left(\frac{\|v\|}{\lambda_{2}}\right)^{\lambda_{1}} \frac{e^{-\left(1-\lambda_{2}\right) r /\|v\|}}{r^{\alpha+\lambda_{1}}}, \quad(r, v) \in \mathbb{R}_{+} \times \mathbb{R}_{0}^{d} .
$$

To prove the claim for $\left\{X_{4, n}\right\}_{n \in \mathbb{N}}$, consider the Lévy measure

$$
v_{(\mathrm{s})}(B)=\int_{\mathbb{R}_{0}^{d}} \int_{\mathbb{R}_{+}} \mathbb{1}_{B}(r v) \frac{1}{r^{\alpha+1}} d r \rho(d v), \quad B \in \mathscr{B}\left(\mathbb{R}_{0}^{d}\right),
$$

which is a non-tempered counterpart of the tempered stable Lévy measure $v$. By a simple change of variables, we have for each $B \in \mathscr{B}\left(\mathbb{R}_{0}^{d}\right)$,

$$
\begin{aligned}
v(B) & =\int_{\mathbb{R}_{0}^{d}} \int_{\mathbb{R}_{+}} \mathbb{1}_{B}\left(r \frac{v}{\|v\|}\right) \frac{e^{-r /\|v\|}}{r^{\alpha+1}} d r m_{\alpha, \rho} \widetilde{\rho}(d v), \\
v_{(\mathrm{s})}(B) & =\int_{\mathbb{R}_{0}^{d}} \int_{\mathbb{R}_{+}} \mathbb{1}_{B}\left(r \frac{v}{\|v\|}\right) \frac{1}{r^{\alpha+1}} d r m_{\alpha, \rho} \widetilde{\rho}(d v),
\end{aligned}
$$

which implies that the Lévy measure $v_{(\mathrm{s})}$ is of a stable law. It is well known that through the inverse Lévy measure method [13, 27], a stable law induced by the Lévy measure $v_{(\mathrm{s})}$ admits a series representation with the kernel $H_{(\mathrm{s})}(r) v /\|v\|$. Clearly, the Lévy measure $v$ is absolutely continuous with respect to $v_{(\mathrm{s})}$ with Radon-Nykodym derivative

$$
\frac{d v}{d v_{(\mathrm{s})}}(r, v)=e^{-r /\|v\|}, \quad(r, v) \in \mathbb{R}_{+} \times \mathbb{R}_{0}^{d} .
$$

By applying the rejection method of Rosiński [33], we get the desired series representation.

For $\left\{X_{5, n}\right\}_{n \in \mathbb{N}}$, it is clear that the kernel $H_{5}(r, v)$ corresponds to the inverse Lévy measure method.

Finally, we prove the infinite divisibility of $\mathscr{L}\left(X_{k, n}\right)$. The truncation scheme $\left\{k \in \mathbb{N}: \Gamma_{k} \leq n\right\}$ corresponds to the truncation to $(0, n)$ of the integral with respect to the variable $r$ in each decomposition of Lévy measure. It is then clear that for $k=1,2,3,4,5, n \in \mathbb{N}$ and $B \in \mathscr{B}\left(\mathbb{R}_{0}^{d}\right), v_{k, n}(B) \leq v(B)$. Hence, the measure $v_{k, n}$ is well defined as a Lévy measure.

(ii) All the representations $v_{k, n}$ are based on the generalized shot noise method due to (2.7). Therefore, by setting $B=\mathbb{R}_{0}^{d}$ and truncating the integral with respect to the Lebesgue measure $d r$ to the interval $(0, n)$, we get the result.

(iii) Due to the truncation by $\left\{k \in \mathbb{N}: \Gamma_{k} \leq n\right\}$, it holds that for each $k=1,2,3,4,5$, and $n \in \mathbb{N}$, the random vector $X_{k, n}$ has the infinitely divisible law with triplet $\left(0,0, v_{k, n}\right)$, such that for each $B \in \mathscr{B}\left(\mathbb{R}_{0}^{d}\right)$,

$$
\begin{aligned}
& v_{1, n}(B)=\int_{\mathbb{R}_{0}^{d}} \int_{0}^{n} \int_{\mathbb{R}_{+}} \int_{0}^{1} \mathbb{1}_{B}\left(\left[\left(\frac{\alpha r}{m_{\alpha, \rho}}\right)^{-1 / \alpha} \wedge w u^{1 / \alpha}\|v\|\right] \frac{v}{\|v\|}\right) d u e^{-w} d w d r \widetilde{\rho}(d v), \\
& v_{2, n}(B)=\int_{\mathbb{R}_{0}^{d}} \int_{0}^{n} \int_{\mathbb{R}_{+}} \mathbb{1}_{B}\left(w \mathbb{1}\left(m_{\alpha, \rho} \frac{\|v\|}{\lambda} \frac{e^{-\frac{1-\lambda}{\|v\|} w}}{w^{\alpha+1}}>r\right) \frac{v}{\|v\|}\right) f(w ; 1, \lambda /\|v\|) d w d r \widetilde{\rho}(d v), \\
& v_{3, n}(B)=\int_{\mathbb{R}_{0}^{d}} \int_{0}^{n} \int_{\mathbb{R}_{+}} \mathbb{1}_{B}\left(w \mathbb{1}\left(m_{\alpha, \rho} \Gamma\left(\lambda_{1}\right)\left(\frac{\|v\|}{\lambda_{2}}\right)^{\lambda_{1}} \frac{e^{-\frac{1-\lambda_{2}}{\|v\|} w}}{w^{\alpha+\lambda_{1}}}>r\right) \frac{v}{\|v\|}\right) \\
& v_{4, n}(B)=\int_{\mathbb{R}_{0}^{d}} \int_{0}^{n} \int_{0}^{1} \mathbb{1}_{B}\left(\left(\frac{\alpha r}{m_{\alpha, \rho}}\right)^{-1 / \alpha} \mathbb{1}\left(e^{-\frac{1}{\|v\|}\left(\frac{\alpha r}{m_{\alpha, \rho}}\right)^{-1 / \alpha}}>u\right) \frac{v}{\|v\|}\right) d u d r \widetilde{\rho}(d v), \\
& v_{5, n}(B)=\int_{\mathbb{R}_{0}^{d}} \int_{0}^{n} \mathbb{1}_{B}\left(H_{5}(r, v) \frac{v}{\|v\|}\right) d r \widetilde{\rho}(d v) .
\end{aligned}
$$


To prove (3.6), it suffices to observe that

$$
\begin{aligned}
\int_{0}^{n} \mathbb{1}_{(x,+\infty)} & \left(\left(\frac{\alpha r}{m_{\alpha, \rho}}\right)^{-1 / \alpha} \wedge w u^{1 / \alpha}\|v\|\right) d r \\
= & \mathbb{1}_{(x,+\infty)}\left(w u^{1 / \alpha}\|v\|\right) \operatorname{Leb}\left(\left\{r \in(0, n):\left(\frac{\alpha r}{m_{\alpha, \rho}}\right)^{-1 / \alpha}>x\right\}\right) \\
= & \mathbb{1}_{(x,+\infty)}\left(w u^{1 / \alpha}\|v\|\right)\left(n \wedge \frac{m_{\alpha, \rho}}{\alpha} x^{-\alpha}\right),
\end{aligned}
$$

which yields the first result with the help of (3.12). Second, to prove (3.7), observe that

$$
\begin{aligned}
& v_{4, n}((x,+\infty) C) \\
& =\int_{\mathbb{R}_{0}^{d}} \int_{0}^{n} \int_{0}^{1} \mathbb{1}\left(e^{-\frac{1}{\|v\|}\left(\frac{\alpha r}{m_{\alpha, \rho}}\right)^{-1 / \alpha}}>u\right) \mathbb{1}_{(x,+\infty)}\left(\left(\frac{\alpha r}{m_{\alpha, \rho}}\right)^{-1 / \alpha}\right) d u d r \mathbb{1}_{C}\left(\frac{v}{\|v\|}\right) \widetilde{\rho}(d v) \\
& =\int_{\mathbb{R}_{0}^{d}} \int_{0}^{n \wedge \frac{m_{\alpha, \rho}}{\alpha} x^{-\alpha}} e^{-\frac{1}{\|v\|}\left(\frac{\alpha r}{m_{\alpha, \rho}}\right)^{-1 / \alpha}} d r \mathbb{1}_{C}\left(\frac{v}{\|v\|}\right) \widetilde{\rho}(d v) \\
& =\int_{\mathbb{R}_{0}^{d}} \int_{x \vee\left(\frac{\alpha n}{m_{\alpha, \rho}}\right)^{-1 / \alpha}}^{+\infty} m_{\alpha, \rho} \frac{e^{-r /\|v\|}}{r^{\alpha+1}} d r \mathbb{1}_{C}\left(\frac{v}{\|v\|}\right) \widetilde{\rho}(d v) \\
& =\int_{\mathbb{R}_{0}^{d}}\left[\left(\int_{0}^{n} e^{-H_{(\mathrm{s})}(r) /\|v\|} d r\right) \wedge \int_{x}^{+\infty} m_{\alpha, \rho} \frac{e^{-r /\|v\|}}{r^{\alpha+1}} d r\right] \mathbb{1}_{C}\left(\frac{v}{\|v\|}\right) \widetilde{\rho}(d v) \\
& \leq \int_{\mathbb{R}_{0}^{d}}\left[n \wedge \int_{x}^{+\infty} m_{\alpha, \rho} \frac{e^{-r /\|v\|}}{r^{\alpha+1}} d r\right] \mathbb{1}_{C}\left(\frac{v}{\|v\|}\right) \widetilde{\rho}(d v) .
\end{aligned}
$$

The claim (3.7) follows from (3.13). Moreover, the inequality (3.8) holds for $v_{4, n}$ by (3.14) since by the definition of the kernel $H_{5}(r, v)$, we have

$$
v_{5, n}((x,+\infty) C)=\int_{\mathbb{R}_{0}^{d}}\left[n \wedge \int_{x}^{+\infty} m_{\alpha, \rho} \frac{e^{-r /\|v\|}}{r^{\alpha+1}} d r\right] \mathbb{1}_{C}\left(\frac{v}{\|v\|}\right) \widetilde{\rho}(d v) .
$$

For $v_{1, n}$, it holds by (3.6) and (3.12) that

$$
\begin{aligned}
v_{1, n}((x,+\infty) C) & =\int_{\mathbb{R}_{0}^{d}}\left(n \alpha x^{\alpha} \int_{x}^{+\infty} \frac{e^{-r /\|v\|}}{r^{\alpha+1}} d r \wedge \int_{x}^{+\infty} m_{\alpha, \rho} \frac{e^{-r /\|v\|}}{r^{\alpha+1}} d r\right) \mathbb{1}_{C}\left(\frac{v}{\|v\|}\right) \widetilde{\rho}(d v) \\
& \leq \int_{\mathbb{R}_{0}^{d}}\left(n \alpha x^{\alpha} \int_{x}^{+\infty} \frac{1}{r^{\alpha+1}} d r \wedge \int_{x}^{+\infty} m_{\alpha, \rho} \frac{e^{-r /\|v\|}}{r^{\alpha+1}} d r\right) \mathbb{1}_{C}\left(\frac{v}{\|v\|}\right) \widetilde{\rho}(d v) \\
& =v_{5, n}((x,+\infty) C) .
\end{aligned}
$$

Finally, we prove the claim (3.8) for $v_{2, n}$ and $v_{3, n}$. Those decompositions are due to the thinning method. Let $\{F(\cdot, v)\}_{v \in \mathbb{R}_{0}^{d}}$ be a measurable family of probability measures on $\mathbb{R}_{+}$such that for each $v \in \mathbb{R}_{0}^{d}$, the support of $F(\cdot, v)$ is $\mathbb{R}_{+}$. Since for $v \in \mathbb{R}_{0}^{d}$, the measure $m_{\alpha, \rho} e^{-w /\|v\|} / w^{\alpha+1} d w$ on $\mathbb{R}_{+}$is absolutely continuous with respect to $F(d w, v)$, the Radon-Nykodym derivative is well defined, for which we will write $G(w, v)$, 
$(w, v) \in \mathbb{R}_{+} \times \mathbb{R}_{0}^{d}$. Then, it holds that for each $x \in \mathbb{R}_{+}$and $C \in \mathscr{B}\left(S^{d-1}\right)$,

$$
\begin{aligned}
\int_{\mathbb{R}_{0}^{d}} \int_{\mathbb{R}_{+}} \int_{0}^{n} \mathbb{1}(G(w, v)>r) d r \mathbb{1}_{(x,+\infty)}(w) F(d w, v) \mathbb{1}_{C}\left(\frac{v}{\|v\|}\right) \widetilde{\rho}(d v) \\
\quad=\int_{\mathbb{R}_{0}^{d}} \int_{x}^{+\infty}[n \wedge G(w, v)] F(d w, v) \mathbb{1}_{C}\left(\frac{v}{\|v\|}\right) \widetilde{\rho}(d v) \\
\leq \int_{\mathbb{R}_{0}^{d}}\left[n \wedge \int_{x}^{+\infty} m_{\alpha, \rho} \frac{e^{-r /\|v\|}}{r^{\alpha+1}} d r\right] \mathbb{1}_{C}\left(\frac{v}{\|v\|}\right) \widetilde{\rho}(d v)=v_{5, n}((x,+\infty) C),
\end{aligned}
$$

where the inequality holds by the Jensen inequality for the concave function $[n \wedge \cdot]$ and by for each $x \in \mathbb{R}_{+}$ and $v \in \mathbb{R}_{0}^{d}, F((x,+\infty), v) \leq 1$.

(iv) Observe that

$$
\left(v_{5, n}-v_{k, n}\right)((x,+\infty) C) \geq\left(v_{k, n}-v_{5, n}\right)((0, x) C),
$$

which follows from (ii) and (iii). It holds by this inequality that for each $x \in \mathbb{R}_{+}$,

$$
\begin{aligned}
\int_{(x,+\infty) C}\|z\|^{q}\left(v_{5, n}-v_{k, n}\right)(d z) & >x^{q}\left(v_{5, n}-v_{k, n}\right)((x,+\infty) C) \\
& \geq x^{q}\left(v_{k, n}-v_{5, n}\right)((0, x) C) \\
& =\int_{(0, x) C} x^{q}\left(v_{k, n}-v_{5, n}\right)(d z)>\int_{(0, x) C}\|z\|^{q}\left(v_{k, n}-v_{5, n}\right)(d z) .
\end{aligned}
$$

Note that the strict inequalities hold since we have assumed the Lévy measure has no atoms. (In particular, the both hand sides in (3.16) tend to get far apart for a higher q.) Hence, it holds that for each $x \in \mathbb{R}_{+}$,

$$
\begin{aligned}
\int_{\mathbb{R}_{0}^{d}}\|z\|^{q} v_{k, n}(d z) & =\int_{(0, x) C}\|z\|^{q} v_{k, n}(d z)-\int_{(x,+\infty) C}\|z\|^{q}\left(v_{5, n}-v_{k, n}\right)(d z)+\int_{(x,+\infty) C}\|z\|^{q} v_{5, n}(d z) \\
& \leq \int_{(0, x) C}\|z\|^{q} v_{k, n}(d z)-\int_{(0, x) C}\|z\|^{q}\left(v_{k, n}-v_{5, n}\right)(d z)+\int_{(x,+\infty) C}\|z\|^{q} v_{5, n}(d z) \\
& =\int_{\mathbb{R}_{0}^{d}}\|z\|^{q} v_{5, n}(d z) .
\end{aligned}
$$

The proof is complete.

The following corollary provides further useful insight into the way the rejection and the inverse Lévy measure methods simulate tails of Lévy measure. The simplification imposed in (i) is not very restrictive and indeed covers most settings of practical interest.

Corollary 3.2. Consider the same setting of Theorem 3.1

(i) If there exists $c \in \mathbb{R}_{+}$such that $\rho\left(\left\{v \in \mathbb{R}_{0}^{d}:\|v\|=c\right\}\right)=1$, then it holds that for each $n \in \mathbb{N}$ and $x \in \mathbb{R}_{+}$,

$$
v_{5, n}\left((x,+\infty) S^{d-1}\right)=n \wedge v\left((x,+\infty) S^{d-1}\right) .
$$

(ii) It holds that for $x \in \mathbb{R}_{+}$,

$$
v_{4, n}\left((x,+\infty) S^{d-1}\right) \sim n \wedge v\left((x,+\infty) S^{d-1}\right), \quad n \uparrow+\infty .
$$

Proof. (i) This claim is trivial from the representation (3.15). 
(ii) Observe that for each $v \in \mathbb{R}_{0}^{d}$, as $n \uparrow+\infty$,

$$
\int_{\left(\frac{\alpha n}{m_{\alpha, \rho}}\right)^{-1 / \alpha}}^{+\infty} m_{\alpha, \rho} \frac{e^{-r /\|v\|}}{r^{\alpha+1}} d r=\frac{m_{\alpha, \rho}}{\|v\|^{\alpha}} \Gamma\left(-\alpha, \frac{1}{\|v\|}\left(\frac{\alpha n}{m_{\alpha, \rho}}\right)^{-1 / \alpha}\right) \sim n,
$$

where we have used the well known asymptotics $\lim _{x \downarrow 0} \Gamma(s, x) / x^{s}=-1 / s$, with $s<0$. This proves the claim.

To close this section, let us present the tail probability asymptotics of the series. Interestingly, the first jumps of all the five different representations have the same tail probability asymptotics, although the captured tail mass differs for different representations under the same truncation scheme. For $\theta \in \mathbb{R}_{+}$and $k \in \mathbb{N}$, define

$$
\begin{gathered}
p_{1}(\theta ; k):=\mathbb{P}\left(\left\|H_{1}\left(\Gamma_{k}, W_{k}^{(1)}, U_{k}, V_{k}\right)\right\|>\theta\right), \\
p_{2}(\theta ; k):=\mathbb{P}\left(\left\|H_{2}\left(\Gamma_{k}, W_{k}^{(2)}, V_{k}\right)\right\|>\theta\right), \\
p_{3}(\theta ; k):=\mathbb{P}\left(\left\|H_{3}\left(\Gamma_{k}, W_{k}^{(3)}, V_{k}\right)\right\|>\theta\right), \\
p_{4}(\theta ; k):=\mathbb{P}\left(\left\|H_{4}\left(\Gamma_{k}, U_{k}, V_{k}\right)\right\|>\theta\right), \\
p_{5}(\theta ; k):=\mathbb{P}\left(\left\|H_{5}\left(\Gamma_{k}, V_{k}\right)\right\|>\theta\right) .
\end{gathered}
$$

Proposition 3.3. It holds that as $\theta \uparrow+\infty$,

$$
\begin{aligned}
& p_{n}(\theta ; k) \sim \frac{m_{\alpha, \rho}^{k-1}}{\Gamma(k+1) \alpha^{k}} \int_{\mathbb{R}_{0}^{d}}\|v\|^{\alpha} \frac{e^{-\theta /\|v\|}}{\theta^{k \alpha}} \rho(d v), \quad n=1,4, \\
& p_{2}(\theta ; k) \sim \frac{m_{\alpha, \rho}^{k-1}}{\Gamma(k+1) \lambda^{k-1}(k+k \alpha-1)} \int_{\mathbb{R}_{0}^{d}}\|v\|^{\alpha-1+k} \frac{e^{-(k-(k-1) \lambda) \theta /\|v\|}}{\theta^{k \alpha-1+k}} \rho(d v), \\
& p_{3}(\theta ; k) \sim \frac{\left(m_{\alpha, \rho} \Gamma\left(\lambda_{1}\right)\right)^{k-1}}{\Gamma(k+1) \lambda_{2}^{(k-1) \lambda_{1}}} \frac{1}{k \alpha+(k-1) \lambda_{1}} \int_{\mathbb{R}_{0}^{d}}\|v\|^{\alpha+(k-1) \lambda_{1}} \frac{e^{-\left(k-(k-1) \lambda_{2}\right) \theta /\|v\|}}{\theta^{k \alpha+(k-1) \lambda_{1}}} \rho(d v), \\
& p_{5}(\theta ; k) \sim \frac{m_{\alpha, \rho}^{k-1}}{\Gamma(k+1) \alpha^{k}} \int_{\mathbb{R}_{0}^{d}}\|v\|^{\alpha} \frac{e^{-\theta /\|v\|}}{\theta^{k \alpha}} \rho(d v) .
\end{aligned}
$$

In particular, it holds that for each $k=1, \ldots, 5$, as $\theta \uparrow+\infty$,

$$
p_{k}(\theta ; 1) \sim \int_{\mathbb{R}_{0}^{d}}\|v\|^{\alpha} \frac{e^{-\theta /\|v\|}}{\alpha \theta^{\alpha}} \rho(d v) .
$$

Proof. Throughout, we will use the notations (3.10) and (3.11) and the asymptotics; for $\beta>0$ and $k \in \mathbb{N}$,

$$
\begin{gathered}
\int_{x}^{+\infty} \frac{e^{-r}}{r^{\beta+1}} d r \sim \frac{e^{-x}}{\beta x^{\beta}}, \quad \text { as } \quad x \uparrow+\infty, \\
\frac{1}{\Gamma(k)} \int_{0}^{x} r^{k-1} e^{-r} d r \sim \frac{x^{k}}{\Gamma(k+1)}, \quad \text { as } \quad x \downarrow 0 .
\end{gathered}
$$


First, it holds by the independence of $\Gamma_{k}, W_{k}$ and $U_{k}$ that

$$
\begin{aligned}
p_{1}(\theta ; k) & =\mathbb{P}\left(\left(\frac{\alpha \Gamma_{k}}{m_{\alpha, \rho}}\right)^{-1 / \alpha}>\theta\right) \mathbb{P}\left(W_{1} U_{1}^{1 / \alpha}\left\|V_{1}\right\|>\theta\right) \\
& =\int_{0}^{\frac{m_{\alpha, \rho}}{\alpha \theta^{\alpha}}} f(y ; k, 1) d y \int_{\mathbb{R}_{0}^{d}} \int_{0}^{1} e^{-\theta u^{-1 / \alpha} /\|v\|} d u \widetilde{\rho}(d v) \\
& =\int_{0}^{\frac{m_{\alpha, \rho}}{\alpha \theta^{\alpha}}} f(y ; k, 1) d y \frac{\alpha \theta^{\alpha}}{m_{\alpha, \rho}} \int_{\mathbb{R}_{0}^{d}} \int_{\theta /\|v\|}^{+\infty} \frac{e^{-s}}{s^{\alpha+1}} d s \rho(d v) \\
& \sim \frac{m_{\alpha, \rho}^{k-1}}{\Gamma(k+1) \alpha^{k}} \int_{\mathbb{R}_{0}^{d}}\|v\|^{\alpha} \frac{e^{-\theta /\|v\|}}{\theta^{k \alpha}} \rho(d v),
\end{aligned}
$$

as $\theta \uparrow+\infty$, which yields the result (3.17) for $n=1$. Next,

$$
\begin{aligned}
p_{2}(\theta ; k) & =\mathbb{P}\left(\left\{W_{1}^{(2)}>\theta\right\} \cap\left\{\Gamma_{k} \leq m_{\alpha, \rho} \frac{\left\|V_{1}\right\|}{\lambda} \frac{e^{-\frac{1-\lambda}{\|v\|} W_{1}^{(2)}}}{\left(W_{1}^{(2)}\right)^{\alpha+1}}\right\}\right) \\
& =\int_{\mathbb{R}_{0}^{d}} \int_{\theta}^{+\infty} \int_{0}^{m_{\alpha, \rho} \frac{\|v\|}{\lambda} \frac{e^{-(1-\lambda) w /\|v\|}}{w^{\alpha+1}}} f(y ; k, 1) d y \frac{\lambda}{\|v\|} e^{-\frac{\lambda}{\|v\|} w} d w \widetilde{\rho}(d v) \\
& \sim \frac{m_{\alpha, \rho}^{k-1}}{\Gamma(k+1) \lambda^{k-1}} \int_{\mathbb{R}_{0}^{d}} \int_{\theta}^{+\infty} \frac{e^{-k \frac{1-\lambda}{\|v\|} w}}{w^{k(\alpha+1)}} e^{-\frac{\lambda}{\|v\|} w} d w\|v\|^{k+\alpha-1} \rho(d v) \\
& \sim \frac{m_{\alpha, \rho}^{k-1}}{\Gamma(k+1) \lambda^{k-1}(k+k \alpha-1)} \int_{\mathbb{R}_{0}^{d}}\|v\|^{\alpha-1+k} \frac{e^{-(k-(k-1) \lambda) \theta /\|v\|}}{\theta^{k \alpha-1+k}} \rho(d v),
\end{aligned}
$$

which yields the result (3.18). Then,

$$
\begin{aligned}
p_{3}(\theta ; k) & \\
= & \mathbb{P}\left(\left\{W_{1}^{(3)}>\theta\right\} \cap\left\{\Gamma_{k} \leq m_{\alpha, \rho} \Gamma\left(\lambda_{1}\right)\left(\frac{\|v\|}{\lambda_{2}}\right)^{\lambda_{1}} \frac{e^{-\frac{1-\lambda_{2}}{\left\|V_{1}\right\|} W_{1}^{(3)}}}{\left(W_{1}^{(3)}\right)^{\alpha+\lambda_{1}}}\right\}\right) \\
= & \int_{\mathbb{R}_{0}^{d}} \int_{\theta}^{+\infty} \int_{0}^{m_{\alpha, \rho} \Gamma\left(\lambda_{1}\right)\left(\frac{\left\|V_{1}\right\|}{\lambda_{2}}\right)^{\lambda_{1}} \frac{e^{-\left(1-\lambda_{2}\right) w /\|v\|}}{w^{\alpha+\lambda_{1}}}} f(y ; k, 1) d y f\left(w ; \lambda_{1}, \lambda_{2} /\|v\|\right) d w \widetilde{\rho}(d v) \\
& \sim \frac{\left(m_{\alpha, \rho} \Gamma\left(\lambda_{1}\right)\right)^{k-1}}{\Gamma(k+1) \lambda_{2}^{(k-1) \lambda_{1}}}\left(k-(k-1) \lambda_{2}\right)^{k \alpha+(k-1) \lambda_{1}} \int_{\mathbb{R}_{0}^{d}}\|v\|^{(1-k) \alpha} \int_{\frac{k-(k-1) \lambda_{2}}{\|v\|}}^{+\infty} \frac{e^{-s}}{s^{k \alpha+(k-1) \lambda_{1}+1}} d s \rho(d v) \\
& \sim \frac{\left(m_{\alpha, \rho} \Gamma\left(\lambda_{1}\right)\right)^{k-1}}{\Gamma(k+1) \lambda_{2}^{(k-1) \lambda_{1}}} \frac{1}{k \alpha+(k-1) \lambda_{1}} \int_{\mathbb{R}_{0}^{d}}\|v\|^{\alpha+(k-1) \lambda_{1}} \frac{e^{-\left(k-(k-1) \lambda_{2}\right) \theta /\|v\|}}{\theta^{k \alpha+(k-1) \lambda_{1}}} \rho(d v),
\end{aligned}
$$


as $\theta \uparrow+\infty$, which yields the result (3.19). Next,

$$
\begin{aligned}
p_{4}(\theta ; k) & =\mathbb{P}\left(\left\{H_{(\mathrm{s})}\left(\Gamma_{k}\right)>\theta\right\} \cap\left\{e^{-H_{(\mathrm{s})}\left(\Gamma_{k}\right) /\left\|V_{1}\right\|}>U_{1}\right\}\right) \\
& =\int_{\mathbb{R}_{0}^{d}} \int_{0}^{\frac{m \alpha, \rho}{\alpha \theta^{\alpha}}} e^{-H_{(\mathrm{s})}(r) /\|v\|} f(r ; k, 1) d r \widetilde{\rho}(d v) \\
& =\frac{m_{\alpha, \rho}^{k-1}}{\Gamma(k) \alpha^{k-1}} \int_{\mathbb{R}_{0}^{d}} \int_{\theta /\|v\|}^{+\infty} s^{-\alpha k-1} e^{-s-\frac{1}{\|v\|^{\alpha}} \frac{m_{\alpha, \rho}}{\alpha s^{\alpha}}} d s\|v\|^{\alpha(1-k)} \rho(d v) \\
& \sim \frac{m_{\alpha, \rho}^{k-1}}{\Gamma(k) \alpha^{k-1}} \int_{\mathbb{R}_{0}^{d}} \int_{\theta /\|v\|}^{+\infty} s^{-\alpha k-1} e^{-s} d s\|v\|^{\alpha(1-k)} \rho(d v) \\
& \sim \frac{m_{\alpha, \rho}^{k-1}}{\Gamma(k+1) \alpha^{k}} \int_{\mathbb{R}_{0}^{d}}\|v\|^{\alpha} \frac{e^{-\theta /\|v\|}}{\theta^{k \alpha}} \rho(d v),
\end{aligned}
$$

as $\theta \uparrow+\infty$, which yields the result (3.17) for $v_{4, n}$. Finally,

$$
\begin{aligned}
p_{5}(\theta ; k) & =\mathbb{P}\left(\Gamma_{k} \leq \int_{\theta}^{+\infty} m_{\alpha, \rho} \frac{e^{-s /\left\|V_{1}\right\|}}{s^{\alpha+1}} d s\right) \\
& =\int_{\mathbb{R}_{0}^{d}} \int_{0}^{\int_{\theta}^{+\infty} m_{\alpha, \rho} s^{-\alpha-1} e^{-s /\|v\|} d s} f(y ; k, 1) d y \widetilde{\rho}(d v) \\
& \sim \frac{1}{\Gamma(k+1)} \int_{\mathbb{R}_{0}^{d}}\left(\int_{\theta}^{+\infty} m_{\alpha, \rho} s^{-\alpha-1} e^{-s /\|v\|} d s\right)^{k} \widetilde{\rho}(d v) \\
& \sim \frac{m_{\alpha, \rho}^{k-1}}{\Gamma(k+1) \alpha^{k}} \int_{\mathbb{R}_{0}^{d}}\|v\|^{\alpha} \frac{e^{-k \theta /\|v\|}}{\theta^{k \alpha}} \rho(d v),
\end{aligned}
$$

as $\theta \uparrow+\infty$, which yields the result (3.20).

\section{Numerical Illustration}

Consider the spectrally positive tempered stable Lévy measure

$$
v(d z)=\frac{a 2^{\kappa} \kappa}{\Gamma(1-\kappa)} \frac{e^{-\frac{1}{2} b^{1 / \kappa} z}}{z^{\kappa+1}} d z, \quad z \in \mathbb{R}_{+},
$$

where $a>0, b \geq 0$ and $\kappa \in(0,1)$. The associated Lévy process $\left\{L_{t}^{(\mathrm{ts})}: t \geq 0\right\}$ (without drift) is called the tempered stable subordinator, with characteristic function

$$
\mathbb{E}\left[e^{i y L_{t}^{(\mathrm{ts})}}\right]=\exp \left[t \int_{\mathbb{R}_{+}}\left(e^{i y z}-1\right) v(d z)\right]=\exp \left[t a\left(b-\left(b^{1 / \kappa}-2 i y\right)^{\kappa}\right)\right] .
$$

Its moments can be derived in closed form. In particular, the first two are given by

$$
\mathbb{E}\left[L_{T}^{(\mathrm{ts})}\right]=2 a \kappa b^{\frac{\kappa-1}{\kappa}} T, \quad \operatorname{Var}\left(L_{T}^{(\mathrm{ts})}\right)=4 a \kappa(1-\kappa) b^{\frac{\kappa-2}{\kappa}} T .
$$

The formulation (4.1) is realized by setting $\alpha=\kappa$ and $\rho$ such that

$$
\rho\left(\mathbb{R}_{0}^{d}\right)=\rho\left(\left\{2 b^{-1 / \kappa}\right\}\right)=\frac{a b \kappa}{\Gamma(1-\kappa)},
$$


in the definition (2.2). Note that $m_{\alpha, \rho}=2^{\kappa} a \kappa / \Gamma(1-\kappa)$. First, its marginal density function is known only in an infinite series representation except for the cases $\kappa=1 / 2$ and $\kappa=1 / 3$. The tempered stable subordinator with $\kappa=1 / 2$ is called an inverse Gaussian process. The density function when $\kappa=1 / 3$ is very intricate due to the presence of a Bessel function. (See [31].)

Here, we compare the following four shot noise series representations;

$$
\begin{aligned}
\left\{L_{t}^{(\mathrm{ts})}\right. & : t \in[0, T]\} \\
& \stackrel{\mathscr{L}}{=}\left\{\sum_{k=1}^{+\infty}\left[\left(\frac{\Gamma(1-\kappa)}{a 2^{\kappa}} \frac{\Gamma_{k}}{T}\right)^{-1 / \kappa} \wedge \frac{W_{k}^{(1)} U_{k}^{1 / \kappa}}{b^{1 / \kappa} / 2}\right] \mathbb{1}_{[0, t]}\left(T_{k}\right): t \in[0, T]\right\} \\
& \stackrel{\mathscr{L}}{=}\left\{\sum_{k=1}^{+\infty} W_{k}^{(2)} \mathbb{1}\left(\frac{\Gamma_{k}}{T} \leq \frac{a \kappa 2^{\kappa+1} b^{-1 / \kappa}}{\Gamma(1-\kappa)\left(W_{k}^{(2)}\right)^{\kappa+1}}\right) \mathbb{1}_{[0, t]}\left(T_{k}\right): t \in[0, T]\right\} \\
& \stackrel{\mathscr{L}}{=}\left\{\sum_{k=1}^{+\infty}\left(\frac{\Gamma(1-\kappa)}{a 2^{\kappa}} \frac{\Gamma_{k}}{T}\right)^{-1 / \kappa} \mathbb{1}\left(e^{-\frac{1}{2} b^{1 / \kappa}\left(\frac{\Gamma(1-\kappa)}{a 2^{\kappa}} \frac{\Gamma_{k}}{T}\right)^{-1 / \kappa}}>U_{k}\right) \mathbb{1}_{[0, t]}\left(T_{k}\right): t \in[0, T]\right\} \\
& \stackrel{\mathscr{L}}{=}\left\{\sum_{k=1}^{+\infty} H_{5}\left(\frac{\Gamma_{k}}{T}, 2 b^{-1 / \kappa}\right) \mathbb{1}_{[0, t]}\left(T_{k}\right): t \in[0, T]\right\} .
\end{aligned}
$$

Recall that $\left\{W_{k}^{(1)}\right\}_{k \in \mathbb{N}}$ is a sequence of iid standard exponential random variables, $\left\{U_{k}\right\}_{k \in \mathbb{N}}$ is a sequence of iid uniform random variables on $[0,1],\left\{W_{k}^{(2)}\right\}_{k \in \mathbb{N}}$ is a sequence of iid exponential random variables with rate $b^{1 / \kappa} / 2$, and $\left\{T_{k}\right\}_{k \in \mathbb{N}}$ is a sequence of iid uniform random variables on $[0, T]$. The kernel $H_{5}(r, v)$ is not available in closed form due to the incomplete gamma function. We employ a numerical approach developed in [18] to obtain this kernel. (For the reader's convenience, we provide its brief description in Appendix $\mathrm{A}$ ) Note that the representation (4.5) can be recovered either from (3.2) with $\lambda=1$, or from (3.3) with $\lambda_{1}=\lambda_{2}=1$.

First, we compare convergence of the representations (4.4)-(4.7) through estimation of $\mathbb{E}\left[L_{T}^{(\mathrm{ts})}\right]$ and $\operatorname{Var}\left(L_{T}^{(\mathrm{ts})}\right)$. Note that the random sequence $\left\{T_{k}\right\}_{k \in \mathbb{N}}$ is not required for this experiment. (Let us remind that series representations can be used for pathwise simulation purpose, not only for computation of expectations.) To avoid overloading the paper with exhaustive numerical results, we only provide results with the parameter setting $(a, b, \kappa, T)=(10,1.6,0.2,1.0)$.

To obtain a (nearly) complete estimator convergence, we generate a sufficiently large number $N=2^{19}(=$ 524288) of iid (truncated) infinite sums on the right hand side of (4.4)-(4.7). We report relative error $\mid\left(\widetilde{\mu}_{n}-\right.$ $\mu) / \mu \mid$ in percentage where $\widetilde{\mu}_{n}$ is a Monte Carlo estimate based on $N$ iid replications and the finite truncation $\left\{k \in \mathbb{N}: \Gamma_{k} \leq n\right\}$ for $\mu_{n}:=\mathbb{E}\left[\widetilde{\mu}_{n}\right]$ such that $\lim _{n \uparrow+\infty} \mu_{n}=\mu$. (Recall that the true value $\mu$ is given by (4.3).)

First of all, it is obvious from the results that the representation (4.7) provides an incomparably faster convergence to the true value in the finite truncation $\left\{k \in \mathbb{N}: \Gamma_{k} \leq n\right\}$, than any other representations (4.4)(4.6). Those results fairly support our theoretical results (3.8) and (3.9). It is particularly remarkable that the representation (4.7) achieves a relative error of less than $1.00 \%$ with much smaller truncation. As indicated, the difference becomes more evident for higher moments. (This is indeed obvious from the inequality (3.16).) On the one hand, the representation (4.5) converge at a fast rate when the truncation level $n$ stays small, while slows down as $n$ increases. The representation (4.6), on the other hand, does not behave well until a very large truncation. In particular, most indicators in (4.6) keep being rejected for small $k$ 's. It seems that the representations (4.4) and (4.6) exhibit a similar performance in terms of the number of terms required to reach a relative error of $1 \%$.

Let us close this section with discussing a further possible improvement through the applicability of low-discrepancy sequences to the representations (4.4)-(4.7). As investigated in [17, 18], on the one hand, it is often effective to apply a suitable low-discrepancy sequence to the interarrival exponential times $\left\{E_{k}\right\}_{k \in \mathbb{N}}$ 


\begin{tabular}{|c|c|c|c|c|c|c|c|c|}
\hline \multirow[b]{2}{*}{ Truncation } & \multicolumn{4}{|c|}{$\mathbb{E}\left[L_{T}^{(\mathrm{ts})}\right]$} & \multicolumn{4}{|c|}{$\operatorname{Var}\left(L_{T}^{(\mathrm{ts})}\right)$} \\
\hline & $\begin{array}{c}\text { Minimum } \\
\text { 4.4. }\end{array}$ & $\begin{array}{c}\text { Thinning } \\
4.5\end{array}$ & $\begin{array}{c}\text { Rejection } \\
4.6\end{array}$ & $\begin{array}{c}\text { Inversion } \\
4.7\end{array}$ & $\begin{array}{c}\text { Minimum } \\
\text { (4.4) }\end{array}$ & $\begin{array}{c}\text { Thinning } \\
4.5\end{array}$ & $\begin{array}{c}\text { Rejection } \\
4.6\end{array}$ & $\begin{array}{c}\text { Inversion } \\
\text { (4.7) }\end{array}$ \\
\hline 1 & $94.78 \%$ & $72.29 \%$ & $100.00 \%$ & $60.29 \%$ & $92.85 \%$ & $41.07 \%$ & $100.00 \%$ & $20.55 \%$ \\
\hline 2 & $89.61 \%$ & $56.57 \%$ & $100.00 \%$ & $43.79 \%$ & $85.88 \%$ & $21.85 \%$ & $100.00 \%$ & $9.53 \%$ \\
\hline 3 & $84.37 \%$ & $47.22 \%$ & $100.00 \%$ & $33.48 \%$ & $78.56 \%$ & $13.74 \%$ & $100.00 \%$ & $5.30 \%$ \\
\hline 4 & $79.17 \%$ & $41.05 \%$ & $100.00 \%$ & $26.39 \%$ & $71.54 \%$ & $10.32 \%$ & $100.00 \%$ & $3.25 \%$ \\
\hline 5 & $73.95 \%$ & $36.50 \%$ & $100.00 \%$ & $21.22 \%$ & $64.32 \%$ & $7.45 \%$ & $100.00 \%$ & $2.21 \%$ \\
\hline 10 & $47.84 \%$ & $24.73 \%$ & $99.56 \%$ & $8.62 \%$ & $28.43 \%$ & $3.22 \%$ & $96.75 \%$ & $0.82 \%$ \\
\hline 15 & $24.42 \%$ & $19.38 \%$ & $57.93 \%$ & $4.22 \%$ & $5.36 \%$ & $1.88 \%$ & $18.73 \%$ & $0.66 \%$ \\
\hline 20 & $11.45 \%$ & $16.27 \%$ & $22.35 \%$ & $2.33 \%$ & $0.82 \%$ & $1.49 \%$ & $1.46 \%$ & $0.63 \%$ \\
\hline 25 & $5.68 \%$ & $14.09 \%$ & $9.61 \%$ & $1.40 \%$ & $0.17 \%$ & $0.68 \%$ & $0.17 \%$ & $0.62 \%$ \\
\hline 30 & $3.04 \%$ & $12.66 \%$ & $4.72 \%$ & $0.89 \%$ & $0.38 \%$ & $1.30 \%$ & $0.37 \%$ & $0.62 \%$ \\
\hline 50 & $0.44 \%$ & $9.12 \%$ & $0.61 \%$ & $0.23 \%$ & $0.09 \%$ & $0.46 \%$ & $0.28 \%$ & $0.45 \%$ \\
\hline 100 & $0.12 \%$ & $5.71 \%$ & $0.04 \%$ & $0.00 \%$ & $0.11 \%$ & $0.06 \%$ & $0.36 \%$ & $0.05 \%$ \\
\hline 150 & $0.02 \%$ & $4.49 \%$ & $0.15 \%$ & $0.01 \%$ & $0.02 \%$ & $0.40 \%$ & $0.36 \%$ & $0.05 \%$ \\
\hline 200 & $0.11 \%$ & $3.64 \%$ & $0.04 \%$ & $0.02 \%$ & $0.10 \%$ & $0.28 \%$ & $0.03 \%$ & $0.05 \%$ \\
\hline
\end{tabular}

Table 1: Relative errors in estimation of the mean $\mathbb{E}\left[L_{T}^{(\mathrm{ts})}\right] \simeq 6.104 \mathrm{E}-1$ and the variance $\operatorname{Var}\left(L_{T}^{(\mathrm{ts})}\right) \simeq 9.313 \mathrm{E}-$ 2. The numbers "Truncation" indicate " $n$ " in $\left\{k \in \mathbb{N}: \Gamma_{k} \leq n\right\}$.

in (2.1) since a systematic generation of the lower dimension of the interarrival times tends to contribute to improvements in both precision and convergence when computing expectations from the nature of the shot noise series representation. On the other hand, it has also been found that the presence of additional random elements water down the effectiveness of a low-discrepancy sequence. We have observed from numerical experiments that the representations (4.4)-(4.6) require so many additional random sequences that the application of low-discrepancy sequences increases computing effort without improving the estimator efficiency at all. In addition, the uniformity of low-discrepancy sequences seems to be ruined even further by the indicator functions in the representations (4.5) and (4.6).

\section{Concluding Remarks}

Tempered stable processes combine both the stable and Gaussian trends, which are two stylized features often seen together in time series of complex systems. They are widely used in various fields of application such as, mathematical finance, econometrics and mathematical biology. In particular, such stochastic processes correspond to the so-called truncated Levy flights in physics and econophysics. Infinite shot noise series representation is the only exact simulation method for the tempered stable process with stability index greater than or equal to one and has recently attracted attention for simulation use.

In this paper, we have derived series representations for the tempered stable laws through the thinning, rejection, and inverse Lévy measure methods. Based upon our rigorous comparison among those representations in terms of the tail mass of Lévy measures which can be simulated under a common finite truncation scheme, the representation via the inverse Lévy measure method achieves a much faster convergence in truncation to the infinite sum than all the other representations. Our results are expected to assist simulation study of, for example, the financial time series.

The results of this paper may be applicable to related stochastic processes. In particular, tempered stable processes of rich flexibility can further be extented through fractionalization and their sample paths can also be simulated through the infinite series representation, as investigated in Houdré and Kawai [21]. Due to additional model flexibility, it is natural to expect a better fit of the model to the observed data, though fractionalization might cause a confusion related to multifractability as pointed out in Heyde and Sly [16]. 
We conjecture that our theoretical analysis is at least implicative, though not necessarily directly applicable, to the series representation of such fractional processes.

\section{A Numerical Inverse Lévy Measure Method}

As observed in Section 3 , the kernel $H_{5}(r, v)$ is not available in closed form, precluded by the incomplete gamma function. To materialize the inverse Lévy measure method for the numerical experiment of Section 4 , we have employed an efficient numerical inversion method of [18]. In principle, it is a generalization of a numerical inversion method proposed in [11], for inversion of probability distribution functions. To employ the method of [11] in our framework, we transform a Lévy measure into a probability distribution function. To be more precise, consider the Lévy measure (4.1) which was investigated in Section 4. This Lévy measure is infinite, that is,

$$
\int_{0}^{+\infty} \frac{a 2^{\kappa} \kappa}{\Gamma(1-\kappa)} \frac{e^{-\frac{1}{2} b^{1 / \kappa} z}}{z^{\kappa+1}} d z=: \int_{0}^{+\infty} w(x) d x=+\infty .
$$

To apply quadrature and interpolation techniques, we need to truncate the support $\mathbb{R}_{+}$to a compact, yet sufficiently large, domain $\left[x_{\min }, x_{\max }\right] \subset \mathbb{R}_{+}$. Let $W(x)$ denote the cumulative Lévy measure, that is,

$$
W(x):=\int_{x}^{+\infty} w(z) d z
$$

where the cumulation here runs down from the infinity rather than up the other way, to avoid explosion at the origin. For convenience, we write $w_{\min }$ and $w_{\max }$ for $W\left(x_{\min }\right)$ and $W\left(x_{\max }\right)$, respectively, that is,

$$
w_{\max }:=W\left(x_{\max }\right)=\int_{x_{\max }}^{+\infty} w(z) d z, \quad w_{\min }:=W\left(x_{\min }\right)=\int_{x_{\min }}^{+\infty} w(z) d z .
$$

Note that $w_{\max }<w_{\min }$. To this end, we define functions $F: \mathbb{R}_{+} \rightarrow[0,1]$ and $f: \mathbb{R}_{+} \rightarrow \mathbb{R}_{+} \cup\{0\}$ by

$$
F(x):=\left\{\begin{array}{ll}
0, & \text { if } x \in\left(0, x_{\min }\right), \\
\frac{w_{\min }-W(x)}{w_{\min }-w_{\max },}, & \text { if } x \in\left[x_{\min }, x_{\max }\right], \\
1, & \text { if } x \in\left(x_{\max },+\infty\right),
\end{array} \quad f(x):= \begin{cases}0, & \text { if } x \in\left(0, x_{\min }\right), \\
\frac{w(x)}{w_{\min }-w_{\max },}, & \text { if } x \in\left[x_{\min }, x_{\max }\right], \\
0, & \text { if } x \in\left(x_{\max },+\infty\right)\end{cases}\right.
$$

which act, respectively, as a cumulative distribution function and a probability density function in the method of [11]. In principle, the method of [11] can then be applied to the standardized cumulative Lévy measure $F(x)$ in (A.1).

Now, the method of [11] consists of two components; the initial setup phase and the actual execution phase. In the initial setup phase, the compact domain $\left[x_{\min }, x_{\max }\right]$ is adaptively divided into disjoint subintervals on the basis of approximation error in $F(x)$ produced by the Newton interpolation. This division should be conducted with great care, as in the actual execution phase, we only obtain an approximation of $F^{-1}(u)$ for each sample $u \in(0,1)$ by the Newton interpolation based on the information $\left\{\left(x_{k}, F\left(x_{k}\right)\right)\right\}_{k \in \mathbb{N}}$ of nodes stored during the initial setup phase, in order to reduce a large amount of computational burden. In our framework, a further extra attention should be paid to computation of $F(x)$ near the origin because it necessarily has a steep peak there. For this reason, the lower truncation $x_{\min }$ has to be chosen very carefully and some safety devices should be employed in algorithms to avoid undesired failures caused by this. The actual execution is enhanced by employing an indexed search algorithm, rather than very inefficient ordinary sequential or binary search, when searching an appropriate subinterval in which the sample value sits. It is claimed in [18] based on numerical experiments that computational effort for this numerical inversion method is relatively small compared to overall simulation. For example, in numerical experiments of Section 4 we set $x_{\min }=1.0 \mathrm{E}-7$ and $x_{\max }=10.0$, and then $w_{\min } \simeq 34.2662$ and $w_{\max } \simeq 0.0000$. The initial setup phase divided the compact domain $\left[x_{\min }, x_{\max }\right]$ into 1753 subintervals and only required 0.344 second.

This numerical method requires a certain amount of initial work for its implementation, while it has the potential to provide significant improvements in simulation accuracy and estimator efficiency, as observed in Section 4 Equally important is that this numerical method is model-free and thus requires no adjustment to different Lévy measures for different problems. 


\section{References}

[1] Asmussen, S., Glynn, P.W. (2007) Stochastic Simulation: Algorithms and Analysis, Springer, New York.

[2] Asmussen, S., Rosiński, J. (2001) Approximations of small jumps of Lévy processes with a view towards simulation, Journal of Applied Probability, 38(2) 482-493.

[3] Baeumer, B., Meerschaert, M.M. (2009) Tempered stable Lévy motion and transit super-diffusion, Journal of Computational and Applied Mathematics, 223(10) 2438-2448.

[4] Barndorff-Nielsen, O.E., Shephard, N. (2001) Non-Gaussian Ornstein-Uhlenbeck-based models and some of their uses in financial economics (with discussion), J. R. Statist. Soc. B, 63(2) 167-241.

[5] Benth, F.E., Gorth, M., Kufakunesu, R. (2007) Valuing volatility and variance swaps for a non-Gaussian Ornstein-Uhlenbeck stochastic volatility model, Applied Mathematical Finance, 14(4) 347-363.

[6] Bondesson, L. (1982) On simulation from infinitely divisible distributions, Advances in Applied Probability, 14(4) 855-869.

[7] Carr, P., Geman, H., Madan, D.B., Yor, M. (2002) The fine structure of asset returns: An empirical investigation, Journal of Business, 75, 303-325.

[8] Carr, P., Geman, H., Madan, D.B., Yor, M. (2003) Stochastic volatility for Lévy processes, Mathematical Finance, 13, $345-382$.

[9] Chambers, J.M., Mallows, C.L., Stuck, B.W. (1976) A method for simulating stable random variables, Journal of the American Statistical Association, 71(354) 340-344.

[10] Cohen, S., Rosiński, J. (2007) Gaussian approximation of multivariate Lévy processes with applications to simulation of tempered stable processes, Bernoulli, 13(1) 195-210.

[11] Derflinger, G., Hörmann, W., Leydold, J. (2009) Random variate generation by numerical inversion when only the density is known, ACM Transactions on Modeling and Computer Simulation, 20(4) Article 18.

[12] Devroye, L. (2009) Random variate generation for exponentially and polynomially tilted stable distributions, ACM Transactions on Modeling and Computer Simulation, 19(4) Article 18.

[13] Ferguson, T.S., Klass, M.J. (1972) A representation of independent increment processes with Gaussian components, Annals of Mathematical Statistics, 43(5) 1634-1643.

[14] Figueiredo, A., Gleria, I., Matsushita, R., Da Silva, S. (2003) On the origins of truncated Lévy flights, Physics Letters A, 315(1-2) 51-60.

[15] Gupta, H.M., Campanha, J.R. (1999) The gradually truncated Lévy flight for systems with power-law distributions, Physica A, 268, 231-239.

[16] Heyde, C.C., Sly, A. (2008) A cautionary note on modeling with fractional Lévy flights, Physica A, 387(21) 5024-5032.

[17] Imai, J., Kawai, R. (2010) Quasi-Monte Carlo methods for infinitely divisible random vectors via series representations, SIAM Journal on Scientific Computing, 32(4) 1879-1897.

[18] Imai, J., Kawai, R., Numerical inverse Lévy measure method for infinite shot noise series representation, preprint.

[19] Imai, J., Kawai, R., On Monte Carlo and quasi-Monte Carlo methods for series representation of infinitely divisible laws, Monte Carlo and Quasi-Monte Carlo Methods 2010, H. Wozniakowski, L. Plaskota (Eds.) Springer-Verlag, forthcoming.

[20] Kawai, R. (2006) An importance sampling method based on the density transformation of Lévy processes, Monte Carlo Methods and Applications, 12(2) 171-186.

[21] Houdré, C., Kawai, R. (2006) On fractional tempered stable motion, Stochastic Processes and their Applications, 116(8) 1161-1184.

[22] Kawai, R., Takeuchi, A., Computation of Greeks for asset price dynamics driven by stable and tempered stable processes, Quantitative Finance, doi:10.1080/14697688.2011.589403.

[23] Kawai, R., Masuda, H. (2011) Exact discrete sampling of finite variation tempered stable Ornstein-Uhlenbeck processes, Monte Carlo Methods and Applications, 17(3) 279-300.

[24] Kawai, R., Masuda, H. (2012) Infinite variation tempered stable Ornstein-Uhlenbeck processes with discrete observations, Communications in Statistics - Simulation and Computation, 41(1) 125-139.

[25] Kawai, R., Masuda, H. (2011) On simulation of tempered stable random variates, Journal of Computational and Applied Mathematics, 235(8) 2873-2887.

[26] Koponen, I. (1995) Analytic approach to the problem of convergence of truncated Lévy flights towards the Gaussian stochastic process, Physical Review E, 52, 1197-1199. 
[27] LePage, R. (1980) Multidimensional infinitely divisible variables and processes II, In: Lecture Notes in Mathematics 860, Springer-Verlag, Berlin, New York, Heidelberg, 279-284.

[28] Mantegna, R.N., Stanley, H.E. (1994) Stochastic process with ultraslow convergence to a Gaussian: The truncated Lévy flights, Physical Review Letters, 73, 2946-2949.

[29] Matsushita, R., Rathie, P., Da Silva, S. (2003) Exponentially damped Lévy flights, Physica A, 326, 544-555.

[30] Nakao, H. (2000) Multi-scaling properties of truncated Lévy flights, Phys. Lett. A, 266, 282-289.

[31] Palmer, K.J., Ridout, M.S., Morgan, B.J.T. (2008) Modelling cell generation times by using the tempered stable distribution, Journal of the Royal Statistical Society: Series C (Applied Statistics), 57(4) 379-397.

[32] Podobnik, B., Ivanov, P.Ch., Lee, Y., Stanley, H.E. (2000) Scale-invariant truncated Lévy process, Europhysics Letters, 52(5) 491.

[33] Rosiński, J. (2001) Series representations of Lévy processes from the perspective of point processes, In: Lévy Processes Theory and Applications, Eds. Barndorff-Nielsen, O.-E., Mikosch, T., Resnick, S.I., Birkhäuser, 401-415.

[34] Rosiński, J. (2007) Tempering stable processes, Stochastic Processes and their Applications, 117(6) 677-707.

[35] Sato, K. (1999) Lévy processes and infinitely divisible distributions, Cambridge University Press.

[36] Tweedie, M.C.K. (1984) An index which distinguishes between some important exponential families, In: Statistics: Applications and New Directions: Proc. Indian Statistical Institute Golden Jubilee International Conference (eds. J. Ghosh and J. Roy) 579-604.

[37] Vinogradov, D.V. (2010) Cumulant approach of arbitrary truncated Levy flight, Physica A, 389(24) 5794-5800. 